\title{
Comparison of Long Noncoding RNA and mRNA Expression Profiles in Mesenchymal Stem Cells Derived from Human Periodontal Ligament and Bone Marrow
}

\author{
Rui Dong, ${ }^{1}$ Juan Du, ${ }^{1}$ Liping Wang, ${ }^{1}$ Jinsong Wang, ${ }^{2,3}$ Gang Ding, \\ Songlin Wang, ${ }^{2,3}$ and Zhipeng Fan ${ }^{1}$ \\ ${ }^{1}$ Laboratory of Molecular Signaling and Stem Cells Therapy, Beijing Key Laboratory of Tooth Regeneration \\ and Function Reconstruction, Capital Medical University School of Stomatology, Beijing 100050, China \\ ${ }^{2}$ Molecular Laboratory for Gene Therapy and Tooth Regeneration, Beijing Key Laboratory of Tooth Regeneration \\ and Function Reconstruction, Capital Medical University School of Stomatology, Beijing 100050, China \\ ${ }^{3}$ Department of Biochemistry and Molecular Biology, Capital Medical University School of Basic Medical Sciences, \\ Beijing 100069, China \\ ${ }^{4}$ Department of Stomatology, Yidu Central Hospital, Weifang Medical University, No. 4138 Linglong Mountain South Road, \\ Qinzhou 262500, China
}

Correspondence should be addressed to Zhipeng Fan; zpfan@ccmu.edu.cn

Received 7 January 2014; Accepted 16 February 2014; Published 27 March 2014

Academic Editor: Jinhua Yu

Copyright (C) 2014 Rui Dong et al. This is an open access article distributed under the Creative Commons Attribution License, which permits unrestricted use, distribution, and reproduction in any medium, provided the original work is properly cited.

\begin{abstract}
Mesenchymal stem cells (MSCs) in different anatomic locations possess diverse biological activities. Maintaining the pluripotent state and differentiation depend on the expression and regulation of thousands of genes, but it remains unclear which molecular mechanisms underlie MSC diversity. Thus, potential MSC applications are restricted. Long noncoding RNAs (lncRNAs) are implicated in the complex molecular circuitry of cellular processes. We investigated differences in lncRNA and mRNA expression profiles between bone marrow stem cells (BMSCs) and periodontal ligament stem cells (PDLSCs) with lncRNA microarray assays and bioinformatics analysis. In PDLSCs, numerous $\operatorname{lncRNAs}$ were significantly upregulated $(n=457)$ or downregulated $(n=513)$ compared to BMSCs. Furthermore, 1,578 mRNAs were differentially expressed. These genes implicated cellular pathways that may be associated with MSC characteristics, including apoptosis, MAPK, cell cycle, and Wnt signaling pathway. Signal-net analysis indicated that phospholipase $\mathrm{C}$ beta 4, filamin B beta, calcium/calmodulin-dependent protein kinase II gamma, and the ionotropic glutamate receptor, AMPA 1, had the highest betweenness centrality among significant genes in the differential gene profile network. A comparison between the coding-noncoding gene coexpression networks of PDLSCs and BMSCs identified chemokine (C-X-C motif) ligand 12 as a core regulatory factor in MSC biology. These results provided insight into the mechanisms underlying MSC biology.
\end{abstract}

\section{Introduction}

Stem cells are undifferentiated cells that can either self-renew or differentiate to produce mature progeny cells $[1,2]$. The two major categories are embryonic and adult stem cells. Adult stem cells are undifferentiated cells found in specialized tissues and organs of adults. Compared to embryonic stem cells, adult stem cells that exist in various organs of the body are easily accessible, and their use is less controversial in terms of ethics $[3,4]$. Mesenchymal stem cells (MSCs) have been identified as mesoderm-derived stromal cells that can differentiate into various mesoderm-type cell lineages. MSCs hold significant promise for tissue regeneration, due to their potential for self-renewal and multilineage differentiation [57]. Humans have abundant adult MSCs available for use in cell-based tissue engineering. MSCs from various tissues, including bone marrow, periosteum, skeletal muscle, and adipose tissue, have similar epitope profiles, but significant 
differences have been observed in MSC properties; that is, MSCs vary in their differentiation, proliferation, and migration potentials according to the tissue source [8-12]. Traditionally, bone-marrow-derived MSCs (BMSCs) have been studied for bone regeneration applications. BMSCs are a population of multipotent, nonhematopoietic marrowderived cells that are easily expanded in culture and differentiate into cells with an osteogenic phenotype $[13,14]$. BMSC transplantations have enhanced periodontal tissue regeneration and bone formation $[15,16]$. Interestingly, $\mathrm{Hu}$ and colleagues investigated whether BMSCs might give rise to different types of epithelial cells, and they tested their potential for serving as a source of ameloblasts. Those results showed, for the first time, that BMSCs could be reprogrammed to become ameloblast-like cells [17]. Thus, BMSCs offered a novel approach for tooth-tissue engineering; they could be induced to become both mesenchymal and epithelial cells in tooth applications [17]. However, scientists disagree on whether BMSCs are ideal seeding cells for tooth engineering. Jing pointed out that the differentiation ability of BMSCs decreases significantly with increasing age of the donor [18]. In the past few decades, several new populations of MSCs have been isolated from dental and craniofacial tissues on the basis of their stem cell properties. These new populations included stem cells derived from the periodontal ligament (PDLSCs), from dental pulp, and from apical papilla, among others [19-24]. When transplanted into animals, these dental tissue-derived stem cells could generate bone/dentin-like mineralized tissue, and they were capable of repairing tooth defects and regenerating periodontal tissue $[21,25,26]$. In contrast to BMSCs, these cells were easily accessible, and they were more intimately associated with dental tissues [3]. Although dental tissue-derived MSCs and BMSCs are regulated by similar factors and share a common protein expression profile, these populations differ significantly in their proliferative ability and developmental potentials in vitro. Furthermore, importantly, they differ in their ability to develop into distinct tissues representative of the microenvironments from which they were derived in vivo. For example, BMSCs formed only bone tissue in the mouse model when treated in the same manner as the dental tissue-derived stem cells $[19,27]$. However, the chondrogenic and adipogenic potentials of dental tissue-derived MSCs appeared to be weaker than those of BMSCs $[22,28]$. Conversely, the neurogenicity of dental tissue-derived stem cells may be more potent than that of BMSCs, probably due to their neural crest origin $[22,28]$.

From the time that dental stem cells were first identified, they have been spotlighted in the dental tissue engineering field. Recently, numerous investigators have attempted to use these cells for dental tissue regeneration and assess their potential in preclinical applications $[26,29]$. However, little is known about the characteristics of dental stem cells and the molecular mechanism underlying their diverse biological activities; thus, their potential application is restricted. Clues on the molecules that control MSC biology can be obtained by comparing molecular expression in MSCs with different biological activities. The development of microarray methods for large-scale analyses of mRNA gene expression has made it possible to search systematically for key molecules [30, 31]. With the introduction of these genome-wide research techniques, various groups have attempted to describe and compare the gene expression patterns of specialized adult stem cells [32-34]. Long, noncoding RNAs (lncRNAs) are transcribed RNA molecules longer than 200 nucleotides. LncRNAs have been shown to have comprehensive functions in both normal development and disease states [35]. Many studies have revealed that lncRNAs exert important roles in biological processes, including roles in cell differentiation, transcription, imprinting, chromatin modification, and others $[36,37]$. Specifically, previous studies have demonstrated that lncRNAs are extremely important for controlling cell or tissue differentiation [38-40].

In this study, we investigated differences in lncRNA and mRNA expression profiles between PDLSCs and BMSCs with microarray assays and bioinformatics analyses. Our results provided useful information for elucidating the different mechanisms that govern MSCs derived from different tissues.

\section{Materials and Methods}

2.1. Cell Culture. All research involving human stem cells complied with the International Society for Stem Cell Research "Guidelines for the Conduct of Human Embryonic Stem Cell Research." We collected impacted, third molars with immature roots from 3 healthy male patients (18-20 years old) under approved guidelines set by the Beijing Stomatological Hospital, Capital Medical University, after obtaining informed patient consent. Molars were removed, disinfected with $75 \%$ ethanol, and then washed with PBS. PDLSCs were isolated from each sample, cultured, and identified as previously described [21]. Briefly, PDLSCs were separated from the periodontal ligament in the middle onethird of the root. Then, the tissue was digested in a solution of $3 \mathrm{mg} / \mathrm{mL}$ collagenase type I (Worthington-Biochem, USA) and $4 \mathrm{mg} / \mathrm{mL}$ dispase (Roche, Germany) for $1 \mathrm{~h}$ at $37^{\circ} \mathrm{C}$. Single-cell suspensions were obtained by passing the cells through a $70 \mu \mathrm{m}$ strainer (Falcon, BD Labware, USA). Three separate PDLSC cultures were grown in a humidified, 5\% $\mathrm{CO}_{2}$ incubator at $37^{\circ} \mathrm{C}$ in alpha-modified Eagle's medium $(\alpha$ MEM; Invitrogen, California, USA) supplemented with $15 \%$ fetal bovine serum (FBS; Invitrogen), $2 \mathrm{mmol} / \mathrm{L}$ glutamine, $100 \mathrm{U} / \mathrm{mL}$ penicillin, and $100 \mu \mathrm{g} / \mathrm{mL}$ streptomycin (Invitrogen).

BMSCs derived from 18-20-year-old males $(n=3)$ were obtained from Cyagen Biosciences (Guangzhou, China). Three separate BMSC cultures were grown in a humidified, $5 \% \mathrm{CO}_{2}$ incubator at $37^{\circ} \mathrm{C}$, in Dulbecco's MEM (Invitrogen), supplemented with $15 \%$ FBS (Invitrogen), $2 \mathrm{mmol} / \mathrm{L}$ glutamine, $100 \mathrm{U} / \mathrm{mL}$ penicillin, and $100 \mu \mathrm{g} / \mathrm{mL}$ streptomycin (Invitrogen). The culture medium was changed every 3 days. All MSCs were used in subsequent experiments after 3-5 passages.

2.2. Microarray Detection. MSCs were grown to $90 \%$ confluence; then, the BMSCs $(n=3)$ and PDLSCs $(n=3)$ were briefly rinsed with PBS, lysed, and total RNA was 
TABLE 1: Primer sequences used in the real-time RT-PCR validation of microarray analyses.

\begin{tabular}{|c|c|c|c|}
\hline Target gene symbol & Primer sequences $\left(5^{\prime}-3^{\prime}\right)$ & Target size (bp) & $T_{m}\left({ }^{\circ} \mathrm{C}\right)$ \\
\hline NR_045555-F & GTTGCAAGGAAACCTTTGGA & \multirow{2}{*}{96} & \multirow{2}{*}{60} \\
\hline NR_045555-R & CTGCATGCTGTTGACCTTGT & & \\
\hline NR_027621-F & CTGCGTGGATTGCTACAAGA & \multirow{10}{*}{102} & \multirow{2}{*}{60} \\
\hline NR_027621-R & CCTTCATAGGCCACCACACT & & \\
\hline XR_111050-F & ATGGCCAGTTCGTTTCTCAC & & \multirow{2}{*}{60} \\
\hline XR_111050-R & AAGACACGTCCTTGGTTTGG & & \\
\hline NR_037595-F & CCCTGTGCAAGAGCACATAA & & \multirow{2}{*}{60} \\
\hline NR_037595-R & TGCCAGCTCATACAAGATGC & & \\
\hline NR_033651-F & CCCCTTGGTATTCTCCCAAT & & \multirow{2}{*}{60} \\
\hline NR_033651-R & CAGCCTTTTGTTGGGTGTTT & & \\
\hline NR_037182-F & CTTCTGCAGGAGGAATCCAG & & \multirow{2}{*}{60} \\
\hline NR_037182-R & TCCCAGTTTTTGGTGACTCC & & \\
\hline GAPDH-F & CGGACCAATACGACCAAATCCG & \multirow{2}{*}{83} & \multirow{2}{*}{60} \\
\hline GAPDH-R & AGCCACATCGCTCAGACACC & & \\
\hline HOXA9-F & CGGTTATGGCATTAAACCTGAACCG & \multirow{2}{*}{67} & \multirow{2}{*}{60} \\
\hline HOXA9-R & GTGAGTGTCAAGCGTGGGACAG & & \\
\hline HOXC8-F & CGGTAAGTTCCAAGGTCTGATACCG & \multirow{2}{*}{99} & \multirow{2}{*}{60} \\
\hline HOXC8-R & CGTCTCCCAGCCTCATGTTTC & & \\
\hline WNT2B-F & СTTTCCTTTGCACCAGCTTC & \multirow{2}{*}{52} & \multirow{2}{*}{60} \\
\hline WNT2B-R & TACССТTCСТCTTGCACACC & & \\
\hline BARX1-F & CGCTTCGAGAAGCAGAAGTA & \multirow{2}{*}{111} & \multirow{2}{*}{60} \\
\hline BARX1-R & CTTCATCCTCCGATTCTGGT & & \\
\hline IGFBP5-F & GCACCTGAGATGAGACAGGA & \multirow{4}{*}{139} & \multirow{2}{*}{60} \\
\hline IGFBP5-R & TGTAGAATCCTTTGCGGTCA & & \\
\hline S100A4-F & GTACTTGGTGTCCACCTTCCACAAGTAC & & \multirow{2}{*}{60} \\
\hline S100A4-R & CCGGGTCAGCAGCTCCTTTAG & & \\
\hline
\end{tabular}

isolated with Trizol reagents (Invitrogen). rRNA was removed from total RNA and purified RNA was amplified and transcribed to produce fluorescent cRNA. Reverse transcription was performed along the entire length of the transcripts, without the $3^{\prime}$ bias, with a random priming method. cDNA was labeled and hybridized to the GeneChip Human Gene 2.0 ST Array (Affymetrix), according to the manufacturer's protocol. After hybridization, washing, and staining, the chip was scanned according to the manufacturer's instructions. Microarray experiments were performed at Genminix Informatic Ltd. (Shanghai, China), a microarray service certified by Affymetrix.

2.3. Real-Time RT-PCR Analysis. Real-time, reverse transcription-PCR (RT-PCR) was used to verify the differential expression of genes that were detected on the microarray. Total RNA was isolated from MSCs with Trizol reagents (Invitrogen). For real-time RT-PCR, $2 \mu \mathrm{g}$ aliquots of RNA as template were combined with random hexamers and reverse transcriptase, according to the manufacturer's protocol (Invitrogen). Real-time PCR reactions were performed with the QuantiTect SYBR Green PCR kit (Qiagen, Germany) and an iCycler iQ Multicolor Real-Time PCR Detection System. The relative level of gene expression was calculated with the $2^{-\Delta \Delta C T}$ method, as previously described [41]. Primers used for amplifying specific genes are shown in Table 1.

2.4. Bioinformatics Analysis. Differentially expressed genes were selected with the TwoClassDif method [9, 42, 43]. Gene ontology (GO) analysis was applied to analyze the main functions of differentially expressed genes. Gene ontology is the key functional classification method used at NCBI. GO can organize genes into hierarchical categories and uncover gene regulatory networks on the basis of biological processes and molecular functions [17, 44]. Based on the Kyoto Encyclopedia of Genes and Genomes (KEGG) database, significantly changed pathways were identified and connected in a pathway network (Path-net), where connections were based on the relationship between these pathways. This approach was previously used to summarize the pathway interactions among genes that were differentially expressed under the influence of disease, and it revealed why certain pathways were activated [45].

Based on the GO and KEGG pathway analyses, we established an interactions repository (Signal-net) derived from KEGG to show the core genes that played an important role in this MSC gene network $[46,47]$. To determine the interactions among genes, we constructed a coding-noncoding gene 
coexpression network (CNC network), which has also been called a gene coexpression network. This CNC network was based on a correlation analysis that evaluated associations between differentially expressed lncRNAs and mRNAs [45]. We calculated the Pearson correlation for each pair of genes and used the most significantly correlated pairs to construct the network [48]. The purpose of network structure analysis was to locate core regulatory factors (genes). In the network, the core regulatory factors were those connected to large numbers of adjacent genes, and, thus, they exhibited the greatest degrees of connectivity. In considering different networks, we evaluated the core regulatory factors by the degree of difference they showed in their roles in the PDLSC and BMSC networks [49], which was measured with the variable Diffk (difference in normalized connectivities).

2.5. Statistics. All statistical calculations were performed with SPSS10 statistical software. Statistical analyses included comparisons with the $t$-test, Fisher's exact test, $\chi^{2}$ test, and the Pearson correlation, as appropriate; $P$ values less than 0.05 were considered statistically significant.

\section{Results}

3.1. Comparison of IncRNA and mRNA Expression Profiles between PDLSCs and BMSCs. To reveal the molecular mechanisms underlying MSCs derived from different tissues, we screened the gene expression patterns in PDLSCs and BMSCs with the human GeneChip microarray method. Because we included only three samples in each group, we applied the RVM $t$-test, which can effectively raise the degrees of freedom in analyses of small sample sizes to filter the genes that were differentially expressed in PDLSCs and BMSCs. After determining significant differences and the false discovery rate (FDR) in the analysis, the differentially expressed genes were selected according to the $P$ value threshold. Hierarchical clustering showed systematic variations in the expression of lncRNAs and mRNAs between PDLSCs and BMSCs. From the microarray data, a comparison of lncRNA expression levels between PDLSCs and BMSCs identified an average of 970 lncRNAs that were significantly differentially expressed (see Supplementary Table 1 in Supplementary Material available online at http://dx.doi.org/10.1155/2014/317853); of those, 457 were upregulated and 513 were downregulated in the PDLSCs compared to the BMSCs. In addition, a total of 1,578 mRNAs were differentially expressed in the PDLSCs and BMSCs (Supplementary Table 2); of those, 862 were upregulated and 716 were downregulated in the PDLSCs compared to the BMSCs.

To confirm the reliability of the microarray data, we randomly selected six lncRNAs among the 970 differentially expressed lncRNAs and analyzed their expression with realtime RT-PCR. These data confirmed that, compared to BMSCs, PDLSCs showed increased expression of the lncRNAs coded as NR_045555, NR_027621, and NR_033651, and decreased expression of the lncRNAs coded as NR_037182, NR_037595, and XR_111050 (Figure 1). Similarly, we randomly selected six mRNAs among the 1,578 differentially expressed
mRNAs and analyzed their expression with real-time RTPCR. These data confirmed that the mRNAs BARX1, S100A4, WNT2B, and IGFBP5 were increased and that the mRNAs HOXA9 and HOXC8 were decreased in PDLSCs compared to BMSCs (Figure 2). The expression levels of these 12 genes were consistent with the microarray results; thus, these results confirmed the reliability of the microarray data.

3.2. Bioinformatics Analysis of BMSC and PDLSC Microarray Data. Next, we performed a bioinformatics analysis to discover the key factors that controlled MSC functions. First, a GO analysis was applied to analyze the main functions of the differentially expressed genes according to gene ontology, which is the key functional classification used by NCBI. According to the threshold, the analysis determined which GOs were significantly differently regulated between PDLSCs and BMSCs with a $P$ value and FDR $<0.05$. The negative logarithm of the $P$ value $(-\operatorname{LgP})$ was used to represent the correlation between gene expression and the relevant biological process. The GO analysis identified 166 genes that were significantly upregulated and 104 that were downregulated among all differentially expressed genes in PDLSCs (data not shown). The results clearly showed which important functions were involved with the differentially expressed genes. The top five upregulated GO functions (upGOs) were related to the response to the mitotic cell cycle, the $M$ phase of the mitotic cell cycle, mitotic prometaphase, the cell cycle checkpoint, and mitotic sister chromatid segregation (Supplementary Figure 1). The top five downregulated GO functions (downGOs) were related to the anterior/posterior pattern, embryonic skeletal system morphogenesis, signal transduction, cochlea morphogenesis, and blood vessel remodeling (Supplementary Figure 2).

Based on the KEGG database, we identified the pathways that mediated the functions of the differentially expressed genes. We identified a total of 67 pathways that showed significant differences due to differential gene expression; changes in 31 pathways involved upregulated genes and changes in 36 pathways involved downregulated genes (Supplementary Figures 3 and 4). We performed Path-net analysis to generate an interaction network that included these significantly changed pathways (Figure 3). The top 3 upregulated pathways were apoptosis, MAPK, and cell cycle signaling. The top 3 downregulated pathways were focal adhesion, Wnt, and adherens junction signaling. In addition, cytokine-cytokine receptor interactions and pathways related to cancer were up/downregulated. These data suggested that these pathways may play key roles in the different core epigenetic mechanisms of PDLSCs and BMSCs.

We performed a Signal-net analysis to further investigate the global network, based on the significantly regulated GOs and pathways. With Signal-net, we screened important candidate genes involved in the differences between PDLSCs and BMSCs (Figure 4). In the Signal-net analysis, the genes are characterized by measuring their "betweenness centrality," the number of times a node is located in the shortest path between 2 other nodes. This measure reflects the importance of a node in a graphic network relative to other 

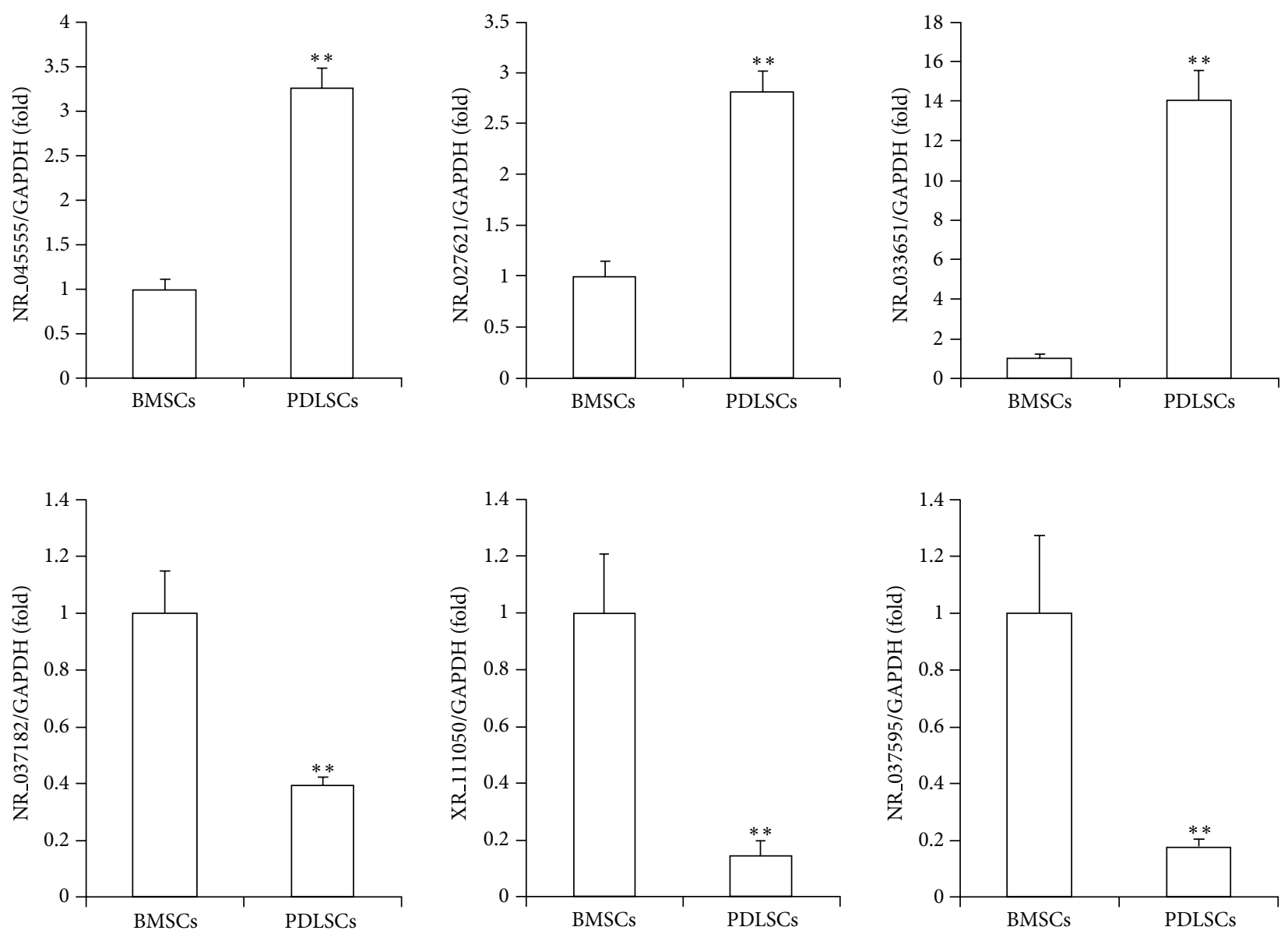

Figure 1: Real-time RT-PCR results show differential lncRNA expression levels in stem cells derived from bone marrow (BMSCs) or periodontal ligament tissue (PDLSCs). The lncRNAs coded as NR_045555, NR_027621, and NR_033651 showed increased expression in PDLSCs, and the lncRNAs coded as NR_037182, XR_111050, and NR_037595 showed decreased expression in PDLSCs compared to BMSCs. GAPDH was used as an internal control. Student's $t$-test was performed to determine statistical significance; all error bars represent s.d. $(n=3$ tissue samples); ${ }^{* *} P<0.01$.

nodes. The four most important differentially expressed genes were identified in the network (Supplementary Table 3); these were phospholipase C beta 4 (PLC $\beta 4$ ), filamin B beta (FLNB), calcium/calmodulin-dependent protein kinase II gamma (CAMK2G), and the ionotropic glutamate receptor, AMPA 1 (GRIAl).

Finally, we used a coding-noncoding gene coexpression (CNC) network to evaluate the interactions among genes and identify the core regulatory genes in the network. Based on our previous results, we built CNC networks to identify the interactions among the differentially expressed lncRNAs and mRNAs in PDLSCs and BMSCs [45]. We used 65 lncRNAs and 208 mRNAs to build the CNC network for PDLSCs and 75 lncRNAs and 187 mRNAs to build the network for BMSCs. In the CNC networks, each mRNA could correlate with one to tens of lncRNAs and vice versa. We used the CNC networks to implicate the interregulation of lncRNAs and mRNAs in the different molecular mechanisms of PDLSCs and BMSCs (Supplementary Figures 5 and 6). In the CNC network of PDLSCs, 17 genes showed a degree $\geq 59$ and a clustering coefficient $\geq 0.6$. This indicated that these genes, including
4 lncRNAs and 13 mRNAs (Table 2), played important roles in the network. In the CNC network of BMSCs, 20 mRNAs showed a degree $\geq 29$ and a clustering coefficient $\geq 0.7$. This indicated that (Table 3 ) these genes played important roles in the network. According to the Diffk values ( $\mid$ Diffk $\mid \geq 0.75$ ) for these networks, 16 genes (Table 4), including 2 lncRNAs and 14 mRNAs, showed different connectivities between PDLSCs and BMSCs, indicating that their roles were different in core pathways that governed MSC functions. The top three mRNAs were chemokine (C-X-C motif) ligand 12 (CXCL12), integrin alpha 2 (ITGA2, CD49B), and cell division cycle 20 homolog (CDC20), which were upregulated. The two lncRNAs identified (|Diffk $\mid \geq 0.75$ ) were FR020479 and FR191603; the former was downregulated and the latter was upregulated.

\section{Discussion}

The presence of different MSCs in dental and craniofacial tissues has encouraged clinical studies to investigate tissue regeneration in orofacial and periodontal regions $[50,51]$. 

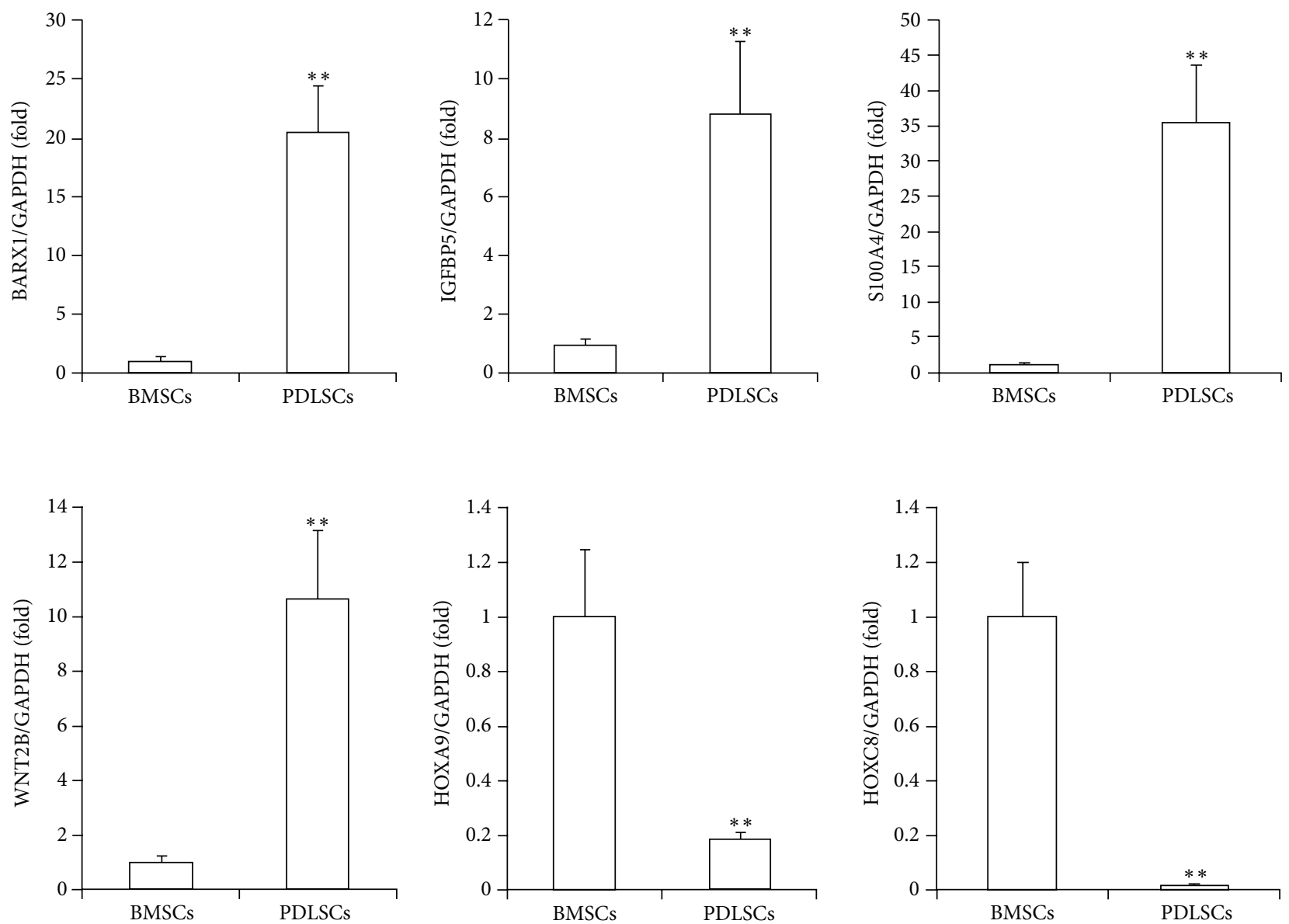

FIGURE 2: Real-time RT-PCR results show differential mRNA expression levels in stem cells derived from bone marrow (BMSCs) or periodontal ligament tissue (PDLSCs). The mRNAs BARX1, IGFBP5, S100A4, and WNT2B showed increased expression, and the mRNAs HOXA9 and HOXC8 showed decreased expression in PDLSCs compared to BMSCs. GAPDH was used as an internal control. Student's $t$-test was performed to determine statistical significance; all error bars represent s.d. $\left(n=3\right.$ tissue samples); ${ }^{* *} P<0.01$.

TABLE 2: Seventeen genes identified in the PDLSC CNC network with high degrees of connectivity and clustering coefficients (degree $\geq 59$, clustering coefficient $\geq 0.6$ ).

\begin{tabular}{|c|c|c|c|c|c|}
\hline Gene symbol & Description & Clustering coefficient & Degree & Style & Type \\
\hline FLNB & Filamin B, beta & 0.67332309 & 63 & Down & mRNA \\
\hline PTTG1 & Pituitary tumor-transforming factor- 1 & 0.72021858 & 61 & Up & mRNA \\
\hline GNG11 & Guanine nucleotide binding protein (G protein), gamma 11 & 0.72021858 & 61 & Up & mRNA \\
\hline IGF1R & Insulin-like growth factor-1 receptor & 0.67431694 & 61 & Up & mRNA \\
\hline ITGA2 & Integrin, alpha 2 (CD49B, alpha 2 subunit of VLA-2 receptor) & 0.66994536 & 61 & Up & mRNA \\
\hline ENPP1 & Ectonucleotide pyrophosphatase/phosphodiesterase 1 & 0.73107345 & 60 & Down & mRNA \\
\hline CDC20 & Cell division cycle 20 homolog (S. cerevisiae) & 0.70734463 & 60 & Up & mRNA \\
\hline COL11A1 & Collagen, type XI, alpha 1 & 0.70734463 & 60 & Down & mRNA \\
\hline DBF4 & DBF4 homolog (S. cerevisiae) & 0.70734463 & 60 & Up & mRNA \\
\hline NR_040093 & gi|338968843|ref|NR_040093.1| & 0.76446523 & 59 & Down & $\operatorname{lncRNA}$ \\
\hline XR_112964 & gi|310115154|ref|XR_112964.1| & 0.74868498 & 59 & Down & $\operatorname{lncRNA}$ \\
\hline XR_108725 & gi|310119896|ref|XR_108725.1| & 0.74868498 & 59 & Down & $\operatorname{lncRNA}$ \\
\hline XR_110624 & gi|310118206|ref|XR_110624.1| & 0.74868498 & 59 & Down & $\operatorname{lncRNA}$ \\
\hline CCNB2 & Cyclin B2 & 0.68322618 & 59 & Up & mRNA \\
\hline GSTM5 & Glutathione S-transferase mu 5 & 0.68322618 & 59 & Up & mRNA \\
\hline HLA-DMA & Major histocompatibility complex, class II, DM alpha & 0.68322618 & 59 & Down & mRNA \\
\hline WASF3 & WAS protein family, member 3 & 0.68264173 & 59 & Down & mRNA \\
\hline
\end{tabular}




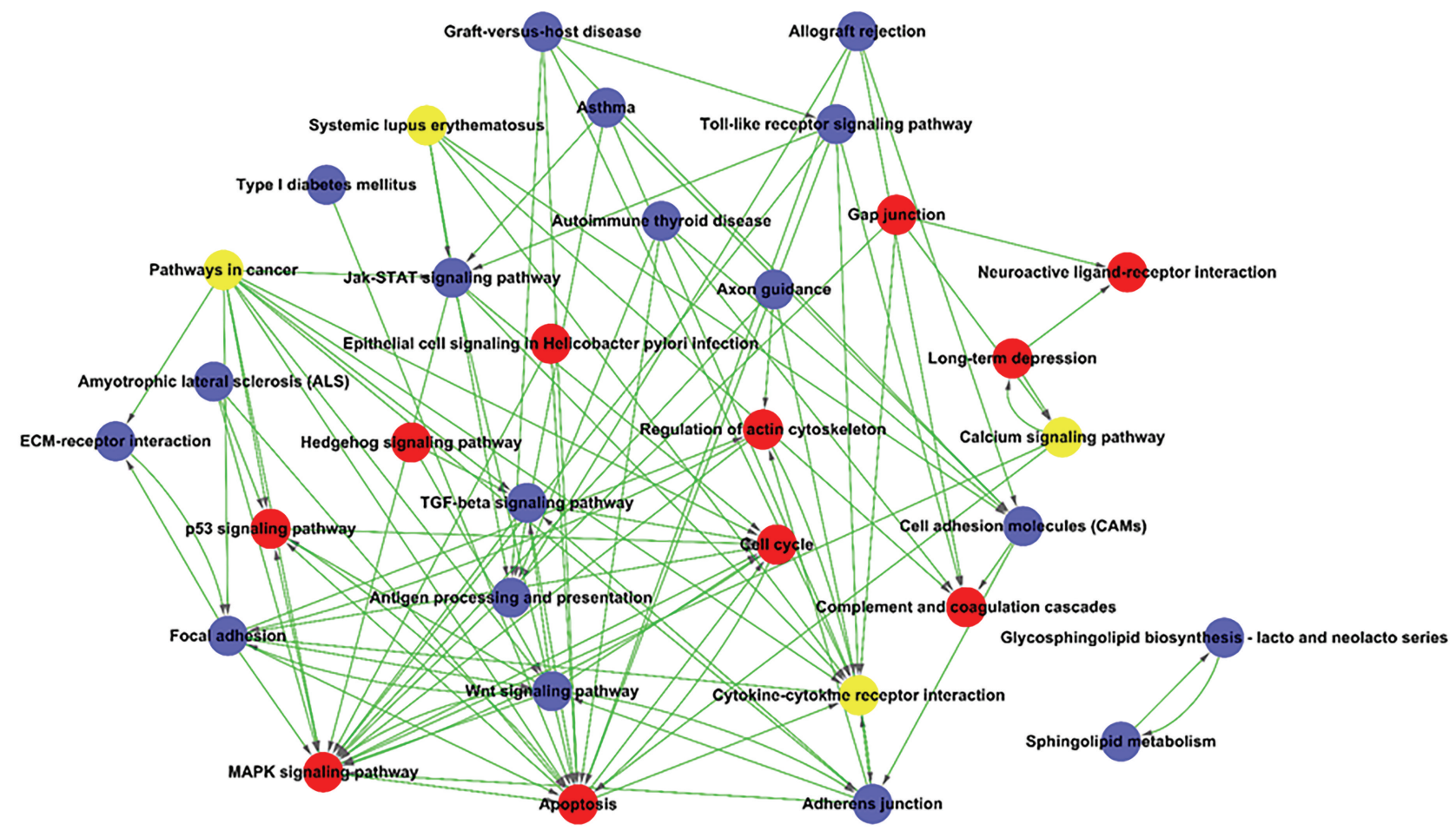

FIGURE 3: The interaction network of significant pathways (Path-net) in stem cells. Pathways that were significantly different between PDLSCs and BMSCs were connected in a Path-net diagram to show the relationships between these pathways. The role of each pathway in the network was measured by counting its connections to upstream and downstream pathways, known as in-degree (upstream connections), out-degree (downstream connections), or degree (all connections). A high degree pathway indicated that it regulated or was regulated by many other pathways, which implied an important role in the signaling network. The circles represent the pathways; blue represents downregulated pathways, red represents upregulated pathways, and yellow represents up- and downregulated pathways. The lines indicate interactions between pathways.

TABle 3: Twenty genes identified in the BMSC CNC network with high degrees of connectivity and clustering coefficients (degree $\geq 29$, clustering coefficient $\geq 0.7)$.

\begin{tabular}{|c|c|c|c|c|c|}
\hline Gene symbol & Description & Clustering coefficient & Degree & Style & Type \\
\hline CXCL12 & Chemokine (C-X-C motif) ligand 12 & 0.75568182 & 33 & Up & mRNA \\
\hline PRIM1 & Primase, DNA, polypeptide 1 (49 kDa) & 0.87931034 & 29 & Up & mRNA \\
\hline LIFR & Leukemia inhibitory factor receptor alpha & 0.87931034 & 29 & Down & mRNA \\
\hline MAD2L1 & MAD2 mitotic arrest deficient-like 1 (yeast) & 0.87931034 & 29 & Up & mRNA \\
\hline TGFBR1 & Transforming growth factor, beta receptor 1 & 0.87931034 & 29 & Down & mRNA \\
\hline PARP1 & Poly(ADP-ribose) polymerase 1 & 0.87931034 & 29 & Up & mRNA \\
\hline FGF5 & Fibroblast growth factor-5 & 0.87931034 & 29 & Up & mRNA \\
\hline CCNE2 & Cyclin E2 & 0.87931034 & 29 & Up & mRNA \\
\hline TTK & TTK protein kinase & 0.87931034 & 29 & Up & mRNA \\
\hline RBL1 & Retinoblastoma-like 1 (p107) & 0.87931034 & 29 & Up & mRNA \\
\hline POLE2 & Polymerase (DNA directed), epsilon 2 (p59 subunit) & 0.87931034 & 29 & Up & mRNA \\
\hline CDK1 & Cyclin-dependent kinase 1 & 0.87931034 & 29 & Up & mRNA \\
\hline MCM3 & Minichromosome maintenance complex component 3 & 0.87931034 & 29 & Up & mRNA \\
\hline CDK2 & Cyclin-dependent kinase 2 & 0.87931034 & 29 & Up & mRNA \\
\hline BMPR1B & Bone morphogenetic protein receptor, type IB & 0.87931034 & 29 & Down & mRNA \\
\hline HIST1H2BO & Histone cluster $1, \mathrm{H} 2 \mathrm{bo}$ & 0.80295567 & 29 & Up & mRNA \\
\hline F10 & Coagulation factor $\mathrm{X}$ & 0.80295567 & 29 & Up & mRNA \\
\hline BDKRB1 & Bradykinin receptor $\mathrm{B} 1$ & 0.80295567 & 29 & Up & mRNA \\
\hline GSTM5 & Glutathione S-transferase mu 5 & 0.80295567 & 29 & Up & mRNA \\
\hline $\mathrm{PRPH} 2$ & Peripherin 2 (retinal degeneration, slow) & 0.80295567 & 29 & Down & mRNA \\
\hline
\end{tabular}




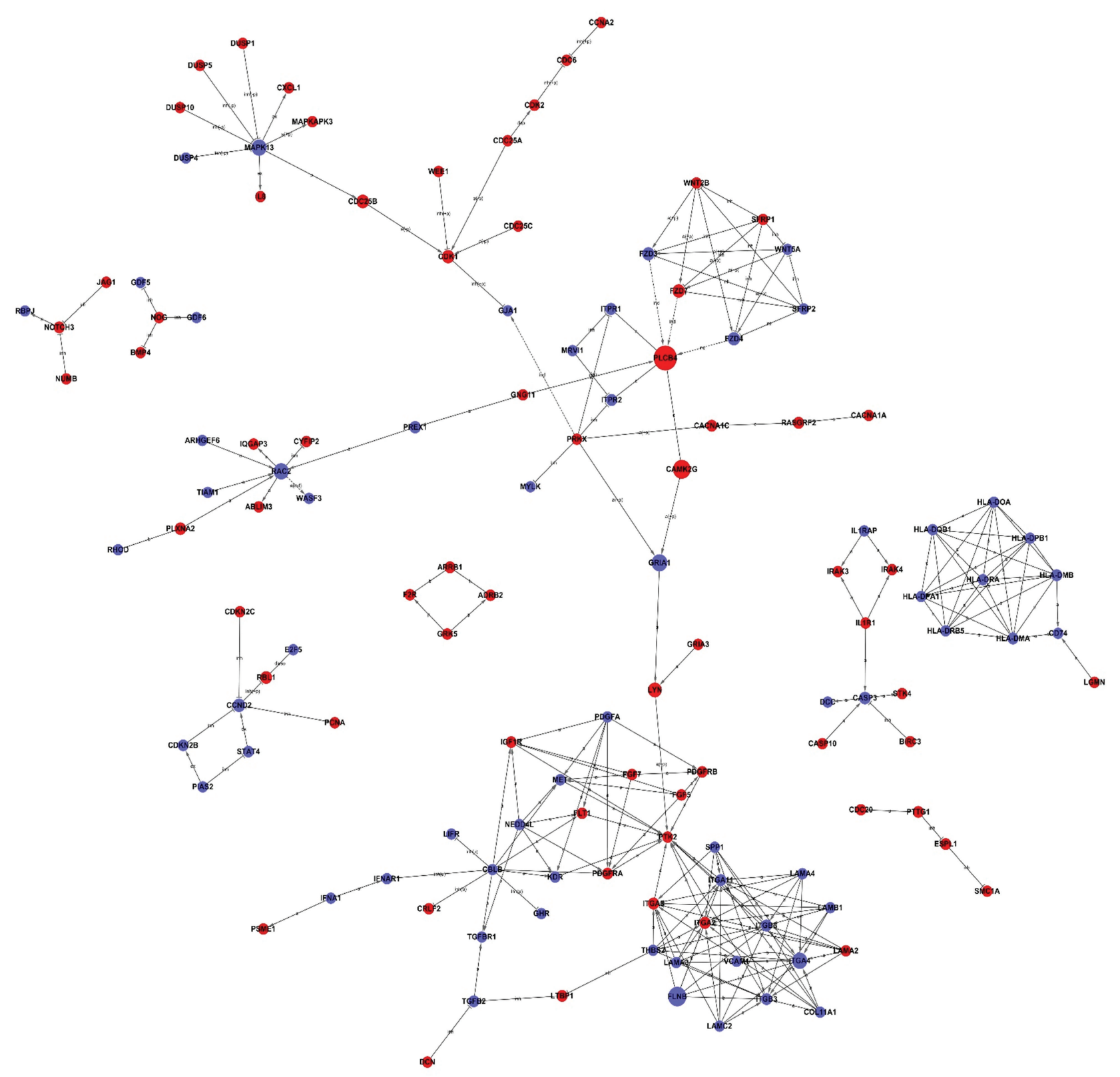

Figure 4: The interaction network of differentially expressed genes (Signal-net). The circles represent important functional genes in PDLSCs (red: upregulated genes; blue: downregulated genes); the circle size represents the degree of interaction (betweenness centrality), and lines indicate the interactions.

In the past few decades, MSC-mediated tissue regeneration has made surprising progress $[25,26,52]$. However, bone marrow has remained the principal source of MSCs for most preclinical and clinical applications. Interestingly, the MSCs from different anatomic locations possess diverse biological activities [8-12]. The challenge lies in identifying the specific genes that are associated with distinct MSC functions. To that end, in the present study, we identified lncRNAs and mRNAs that were differentially expressed in PDLSCs and BMSCs.

We identified 970 differentially expressed lncRNAs and 1,578 differentially expressed mRNAs in BMSCs and dental tissue-derived MSCs. This information may be useful for further studies on gene functions and regulation mechanisms in MSCs. Furthermore, we found that several of the upregulated genes in PDLSCs may be associated with PDLSC characteristics. For instance, $B A R X 1$, a transcription factor expressed in the mesenchyme of molar primordia, is involved in the regulation of tooth morphogenesis, in the development of tooth and craniofacial mesenchyme that originates from the neural crest [53-55], and possibly, in the regulation of MSC differentiation.

To identify the key factors that regulated MSC functions, we applied bioinformatics analyses to classify the microarray data. The GO analysis revealed specific functional pathways 
TABLE 4: Sixteen genes with different pathway connectivities (identified with Diffk) in PDLSCs and BMSCs (|Diffk| $\geq 0.75)$.

\begin{tabular}{|c|c|c|c|c|}
\hline Gene symbol & Description & Style & Type & |Diffk \\
\hline CXCL12 & Chemokine (C-X-C motif) ligand 12 & Up & mRNA & 1 \\
\hline ITGA2 & Integrin, alpha 2 (CD49B, alpha 2 subunit of VLA-2 receptor) & Up & mRNA & 0.968254 \\
\hline CDC20 & Cell division cycle 20 homolog (S. cerevisiae) & Up & mRNA & 0.952381 \\
\hline WASF3 & WAS protein family, member 3 & Down & mRNA & 0.9365079 \\
\hline CAMK4 & Calcium/calmodulin-dependent protein kinase IV & Up & mRNA & 0.8571429 \\
\hline SEMA3C & Sema domain, immunoglobulin domain (Ig), short basic domain, secreted, (semaphorin) 3C & Down & mRNA & 0.8571429 \\
\hline CCNA2 & Cyclin A2 & Up & mRNA & 0.8253968 \\
\hline POLA1 & Polymerase (DNA directed), alpha 1, catalytic subunit & Up & mRNA & 0.8253968 \\
\hline FR020479 & AB209345, AC006512, U47924 & Down & $\operatorname{lncRNA}$ & 0.7878788 \\
\hline FR191603 & AJ609445, AK128061, AP001273 & Up & $\operatorname{lncRNA}$ & 0.7532468 \\
\hline SLK & STE20-like kinase & Up & mRNA & 0.7460317 \\
\hline PDGFA & Platelet-derived growth factor alpha polypeptide & Down & mRNA & 0.7388167 \\
\hline PTK2 & PTK2 protein tyrosine kinase 2 & Up & mRNA & 0.7142857 \\
\hline ENPP1 & Ectonucleotide pyrophosphatase/phosphodiesterase 1 & Down & mRNA & 0.7099567 \\
\hline PRKCE & Protein kinase C, epsilon & Up & mRNA & 0.7056277 \\
\hline BDKRB1 & Bradykinin receptor $\mathrm{B} 1$ & Up & mRNA & 0.7041847 \\
\hline
\end{tabular}

that were enriched in the genes responsible for the divergent features of PDLSCs and BMSCs. These differentially expressed genes were subsequently organized into hierarchical categories based on pertinent biological processes. A high degree pathway interacted with a high number of other pathways, which implied an important role in cell biological features. Further pathway analyses indicated that apoptosis, MAPK, cytokine-cytokine receptor interaction, focal adhesion, pathways in cancer, Wnt, cell cycle, and adherens junctions signaling pathways were involved in the diverse biological activities of PDLSCs and BMSCs. It is well known that these pathways play an important role in regulating cellular apoptosis, survival, and differentiation.

To identify important genes involved in the different epigenetic mechanisms of PDLSCs and BMSCs, we performed Signal-net analysis on the significantly regulated GOs and pathways. This analysis revealed that PLC $\beta 4$, FLNB, CAMK2G, and GRIA1 exhibited the most betweenness centrality. PLC $\beta 4$ and CAMK2G were upregulated in PDLSCs. It was reported that PLC $\beta 4$ was highly expressed in the retina and the cerebellum, where calcium plays an important role in the transduction of extracellular signals [56-58]. Moreover, CAMK2G is activated by intracellular calcium/calmodulin [59]. Thus, the Signal-net analysis results suggested that these genes were important in calcium-sensitive signaling cascades that regulate cell function. In addition, FLNB regulates intracellular communication and signaling by linking the protein actin to the cell membrane. This activity allows direct communication between the cell membrane and the cytoskeletal network, which provides a means to control and guide proper skeletal development $[60,61]$.

The CNC network comparisons indicated that CXCL12 was a core regulatory factor, which may be involved in the diverse biological activities of PDLSCs and BMSCs. CXCL12, also known as stromal cell-derived factor-1, stimulates migration by rearranging the actin cytoskeleton, increasing focal adhesion, and stimulating matrix metalloproteinase production in MSCs $[62,63]$. Thus, CXCL12 can recruit MSC to participate in the regeneration of injured tissues [64]. Presumably, MSC migration is mediated through an intracellular pathway, for example, the MAPK/ERK signaling pathways [62]. Our results were consistent with previous reports and may also be applicable to the differentiation mechanisms previously described in MSCs.

Additionally, we identified some lncRNAs that were differentially expressed in PDLSCs and BMSCs, for example, FR020479 and FR191603. Previous studies demonstrated that IncRNAs may function by controlling the transcriptional regulation of neighboring coding genes $[65,66]$. Identifying differentially expressed nearby coding mRNAs may enhance our understanding of the function of lncRNAs in MSCs. However, further studies must be performed to investigate that hypothesis.

\section{Conclusion}

This study provided comprehensive profiles of mRNA and lncRNA expression in PDLSCs and BMSCs, two tissuederived MSCs. In addition, potential regulatory mechanisms were identified with bioinformatics analyses. Although more studies are required to demonstrate the precise role and mechanisms of these lncRNAs and mRNAs, the genomic data we identified with microarray analyses may increase our understanding of MSC biology.

\section{Conflict of Interests}

The authors declare that there is no potential conflict of interests. 


\section{Acknowledgments}

This work was supported by Grants from the National Natural Science Foundation of China (81271101 to Zhipeng Fan, 81271100 to Rui Dong, and 81170931 to Juan Du), the Program for New Century Excellent Talents in the University (NCET12-0611 to Zhipeng Fan), High-Level Talents of the Beijing Health System (2013-3-035 to Zhipeng Fan and 2013-3-034 to Rui Dong), the Beijing Natural Science Foundation (7112057 to Rui Dong), and the Beijing Nova Program (2011083 to Rui Dong).

\section{References}

[1] J. Cai, M. L. Weiss, and M. S. Rao, "In search of "stemness"' Experimental Hematology, vol. 32, no. 7, pp. 585-598, 2004.

[2] A. J. Wagers and I. L. Weissman, "Plasticity of adult stem cells," Cell, vol. 116, no. 5, pp. 639-648, 2004.

[3] C. Morsczeck, G. Schmalz, T. E. Reichert, F. Völlner, K. Galler, and O. Driemel, "Somatic stem cells for regenerative dentistry," Clinical Oral Investigations, vol. 12, no. 2, pp. 113-118, 2008.

[4] A. Mauron and M. E. Jaconi, "Stem cell science: current ethical and policy issues," Clinical Pharmacology and Therapeutics, vol. 82, no. 3, pp. 330-333, 2007.

[5] B. N. Jahagirdar and C. M. Verfaillie, "Multipotent adult progenitor cell and stem cell plasticity," Stem Cell Reviews, vol. 1, no. 1, pp. 53-59, 2005.

[6] D. G. Phinney and D. J. Prockop, "Concise review: mesenchymal stem/multipotent stromal cells: the state of transdifferentiation and modes of tissue repair-current views," Stem Cells, vol. 25, no. 11, pp. 2896-2902, 2007.

[7] J. Feng, A. Mantesso, C. de Bari, A. Nishiyama, and P. T. Sharp, "Dual origin of mesenchymal stem cells contributing to organ growth and repair," Proceedings of the National Academy of Sciences of the United States of America, vol. 108, no. 16, pp. 65036508, 2011.

[8] H. Yang, L. N. Gao, Y. An et al., "Comparison of mesenchymal stem cells derived from gingival tissue and periodontal ligament in different incubation conditions," Biomaterials, vol. 34, no. 29, pp. 7033-7047, 2013.

[9] S. Yu, J. Long, J. Yu et al., "Analysis of differentiation potentials and gene expression profiles of mesenchymal stem cells derived from periodontal ligament and Wharton's jelly of the umbilical cord," Cells Tissues Organs, vol. 197, no. 3, pp. 209-223, 2013.

[10] O. Hayashi, Y. Katsube, M. Hirose, H. Ohgushi, and H. Ito, "Comparison of osteogenic ability of rat mesenchymal stem cells from bone marrow, periosteum, and adipose tissue," Calcified Tissue International, vol. 82, no. 3, pp. 238-247, 2008.

[11] Y. Sakaguchi, I. Sekiya, K. Yagishita, and T. Muneta, "Comparison of human stem cells derived from various mesenchymal tissues: superiority of synovium as a cell source," Arthritis and Rheumatism, vol. 52, no. 8, pp. 2521-2529, 2005.

[12] Y. Zhang, C.-D. Li, X.-X. Jiang, H.-L. Li, P.-H. Tang, and N. Mao, "Comparison of mesenchymal stem cells from human placenta and bone marrow," Chinese Medical Journal, vol. 117, no. 6, pp. 882-887, 2004.

[13] A. I. Caplan, "Mesenchymal stem cells: cell-based reconstructive therapy in orthopedics," Tissue Engineering, vol. 11, no. 7-8, pp. 1198-1211, 2005.

[14] S. P. Bruder, K. H. Kraus, V. M. Goldberg, and S. Kadiyala, "The effect of implants loaded with autologous mesenchymal stem cells on the healing of canine segmental bone defects," Journal of Bone and Joint Surgery A, vol. 80, no. 7, pp. 985-996, 1998.

[15] Y. M. Lee, Y. J. Seol, Y. T. Lim et al., "Tissue-engineered growth of bone by marrow cell transplantation using porous calcium metaphosphate matrices," Journal of Biomedical Materials Research, vol. 54, pp. 216-223, 2001.

[16] H. Kawaguchi, A. Hirachi, N. Hasegawa et al., "Enhancement of periodontal tissue regeneration by transplantation of bone marrow mesenchymal stem cells," Journal of Periodontology, vol. 75, no. 9, pp. 1281-1287, 2004.

[17] B. Hu, F. Unda, S. Bopp-Kuchler et al., "Bone marrow cells can give rise to ameloblast-like cells," Journal of Dental Research, vol. 85, no. 5, pp. 416-421, 2006.

[18] W. Jing, L. Wu, Y. Lin, L. Liu, W. Tang, and W. Tian, "Odontogenic differentiation of adipose-derived stem cells for tooth regeneration: necessity, possibility, and strategy," Medical Hypotheses, vol. 70, no. 3, pp. 540-542, 2008.

[19] S. Gronthos, M. Mankani, J. Brahim, P. G. Robey, and S. Shi, "Postnatal human dental pulp stem cells (DPSCs) in vitro and in vivo," Proceedings of the National Academy of Sciences of the United States of America, vol. 97, no. 25, pp. 13625-13630, 2000.

[20] M. Miura, S. Gronthos, M. Zhao et al., "SHED: stem cells from human exfoliated deciduous teeth," Proceedings of the National Academy of Sciences of the United States of America, vol. 100, no. 10, pp. 5807-5812, 2003.

[21] B.-M. Seo, M. Miura, S. Gronthos et al., "Investigation of multipotent postnatal stem cells from human periodontal ligament," The Lancet, vol. 364, no. 9429, pp. 149-155, 2004.

[22] W. Sonoyama, Y. Liu, T. Yamaza et al., "Characterization of the apical papilla and its residing stem cells from human immature permanent teeth: a pilot study," Journal of Endodontics, vol. 34, no. 2, pp. 166-171, 2008.

[23] R. d'Aquino, A. Graziano, M. Sampaolesi et al., "Human postnatal dental pulp cells co-differentiate into osteoblasts and endotheliocytes: a pivotal synergy leading to adult bone tissue formation," Cell Death and Differentiation, vol. 14, no. 6, pp. 1162-1171, 2007.

[24] G. Laino, R. D’Aquino, A. Graziano et al., "A new population of human adult dental pulp stem cells: a useful source of living autologous fibrous bone tissue (LAB)," Journal of Bone and Mineral Research, vol. 20, no. 8, pp. 1394-1402, 2005.

[25] G. Ding, Y. Liu, W. Wang et al., "Allogeneic periodontal ligament stem cell therapy for periodontitis in swine," Stem Cells, vol. 28, no. 10, pp. 1829-1838, 2010.

[26] Y. Liu, Y. Zheng, G. Ding et al., "Periodontal ligament stem cellmediated treatment for periodontitis in miniature swine," Stem Cells, vol. 26, no. 4, pp. 1065-1073, 2008.

[27] S. Batouli, M. Miura, J. Brahim et al., "Comparison of stem-cellmediated osteogenesis and dentinogenesis," Journal of Dental Research, vol. 82, no. 12, pp. 976-981, 2003.

[28] W. Zhang, X. F. Walboomers, S. Shi, M. Fan, and J. A. Jansen, "Multilineage differentiation potential of stem cells derived from human dental pulp after cryopreservation," Tissue Engineering, vol. 12, no. 10, pp. 2813-2823, 2006.

[29] S.-H. Kim, K.-H. Kim, B.-M. Seo et al., "Alveolar bone regeneration by transplantation of periodontal ligament stem cells and bone marrow stem cells in a canine peri-implant defect model: a pilot study," Journal of Periodontology, vol. 80, no. 11, pp. 18151823, 2009.

[30] D. J. Duggan, M. Bittner, Y. Chen, P. Meltzer, and J. M. Trent, "Expression profiling using cDNA microarrays," Nature Genetics, vol. 21, no. 1, pp. 10-14, 1999. 
[31] P. O. Brown and D. Botstein, "Exploring the new world of the genome with DNA microarrays," Nature Genetics, vol. 21, no. 1, pp. 33-37, 1999.

[32] S. Shi, P. G. Robey, and S. Gronthos, "Comparison of human dental pulp and bone marrow stromal stem cells by cDNA microarray analysis," Bone, vol. 29, no. 6, pp. 532-539, 2001.

[33] N. Inomata, H. Tomita, Z. Ikezawa, and H. Saito, "Differential gene expression profile between cord blood progenitor-derived and adult progenitor-derived human mast cells," Immunology Letters, vol. 98, no. 2, pp. 265-271, 2005.

[34] T. Schilling, R. Küffner, L. Klein-Hitpass, R. Zimmer, F. Jakob, and N. Schütze, "Microarray analyses of transdifferentiated mesenchymal stem cells," Journal of Cellular Biochemistry, vol. 103, no. 2, pp. 413-433, 2008.

[35] C. P. Ponting, P. L. Oliver, and W. Reik, "Evolution and functions of long noncoding RNAs," Cell, vol. 136, no. 4, pp. 629-641, 2009.

[36] J. T. Kung, D. Colognori, and J. T. Lee, "Long noncoding RNAs: past, present, and future," Genetics, vol. 193, no. 3, pp. 651-669, 2013.

[37] S. Ghosal, S. Das, and J. Chakrabarti, "Long noncoding RNAs: new players in the molecular mechanism for maintenance and differentiation of pluripotent stem cells," Stem Cells and Development, vol. 22, no. 16, pp. 2240-2253, 2013.

[38] M. Cesana, D. Cacchiarelli, I. Legnini et al., "A long noncoding RNA controls muscle differentiation by functioning as a competing endogenous RNA," Cell, vol. 147, no. 2, pp. 358-369, 2011.

[39] W. Hu, J. R. Alvarez-Dominguez, and H. F. Lodish, "Regulation of mammalian cell differentiation by long non-coding RNAs," EMBO Reports, vol. 13, no. 11, pp. 971-983, 2012.

[40] M. Kretz, D. E. Webster, R. J. Flockhart et al., "Suppression of progenitor differentiation requires the long noncoding RNA ANCR," Genes and Development, vol. 26, no. 4, pp. 338-343, 2012.

[41] S. A. Bustin, "Absolute quantification of mrna using real-time reverse transcription polymerase chain reaction assays," Journal of Molecular Endocrinology, vol. 25, no. 2, pp. 169-193, 2000.

[42] G. W. Wright and R. M. Simon, "A random variance model for detection of differential gene expression in small microarray experiments," Bioinformatics, vol. 19, no. 18, pp. 2448-2455, 2003.

[43] R. Clarke, H. W. Ressom, A. Wang et al., "The properties of highdimensional data spaces: implications for exploring gene and protein expression data," Nature Reviews Cancer, vol. 8, no. 1, pp. 37-49, 2008.

[44] M. Ashburner, C. A. Ball, J. A. Blake et al., "Gene ontology: tool for the unification of biology," Nature Genetics, vol. 25, no. 1, pp. 25-29, 2000.

[45] M. A. Pujana, J.-D. J. Han, L. M. Starita et al., "Network modeling links breast cancer susceptibility and centrosome dysfunction," Nature Genetics, vol. 39, no. 11, pp. 1338-1349, 2007.

[46] C. Li and H. Li, "Network-constrained regularization and variable selection for analysis of genomic data," Bioinformatics, vol. 24, no. 9, pp. 1175-1182, 2008.

[47] J. D. Zhang and S. Wiemann, "KEGGgraph: a graph approach to KEGG PATHWAY in R and bioconductor," Bioinformatics, vol. 25, no. 11, pp. 1470-1471, 2009.

[48] C. Prieto, A. Risueño, C. Fontanillo, and J. de Las Rivas, "Human gene coexpression landscape: confident network derived from tissue transcriptomic profiles," PLoS ONE, vol. 3, no. 12, Article ID e3911, 2008.
[49] M. R. J. Carlson, B. Zhang, Z. Fang, P. S. Mischel, S. Horvath, and S. F. Nelson, "Gene connectivity, function, and sequence conservation: predictions from modular yeast co-expression networks," BMC Genomics, vol. 7, article 40, 2006.

[50] K. G. Silvério, B. B. Benatti, M. Z. Casati, E. A. Sallum, and F. H. Nociti Jr., "Stem cells: potential therapeutics for periodontal regeneration," Stem Cell Reviews, vol. 4, no. 1, pp. 13-19, 2008.

[51] L. Rimondini and S. Mele, "Stem cell technologies for tissue regeneration in dentistry," Minerva Stomatologica, vol. 58, no. 10, pp. 483-500, 2009.

[52] A. Giuliani, A. Manescu, M. Langer et al., "Three years after transplants in human mandibles, histological and in-line holotomography revealed that stem cells regenerated a compact rather than a spongy bone: biological and clinical implications," Stem Cells Translational Medicine, vol. 2, no. 4, pp. 316-324, 2013.

[53] S. M. Sperber and I. B. Dawid, "barxl is necessary for ectomesenchyme proliferation and osteochondroprogenitor condensation in the zebrafish pharyngeal arches," Developmental Biology, vol. 321, no. 1, pp. 101-110, 2008.

[54] I. Miletich, G. Buchner, and P. T. Sharpe, "Barxl and evolutionary changes in feeding," Journal of Anatomy, vol. 207, no. 5, pp. 619-622, 2005.

[55] T. A. Mitsiadis, M.-L. Mucchielli, S. Raffo, J.-P. Proust, P. Koopman, and C. Goridis, "Expression of the transcription factors Otlx2, Barx1 and Sox9 during mouse odontogenesis," European Journal of Oral Sciences, vol. 106, supplement 1, pp. 112-116, 1998.

[56] C. W. Taylor, "Controlling calcium entry," Cell, vol. 111, no. 6, pp. 767-769, 2002.

[57] Y. Kido, C. T. Gordon, S. Sakazume et al., "Further characterization of atypical features in auriculocondylar syndrome caused by recessive PLCB4 mutations," American Journal of Medical Genetics A, vol. 161, no. 9, pp. 2339-2346, 2013.

[58] A. M. Lyon and J. J. Tesmer, "Structural insights into phospholipase C-beta function," Molecular Pharmacology, vol. 84, no. 4, pp. 488-500, 2013.

[59] M. Mattingsdal, A. A. Brown, S. Djurovic et al., "Pathway analysis of genetic markers associated with a functional MRI faces paradigm implicates polymorphisms in calcium responsive pathways," Neuroimage, vol. 70, pp. 143-149, 2013.

[60] J. Lu, G. Lian, R. Lenkinski et al., "Filamin B mutations cause chondrocyte defects in skeletal development," Human Molecular Genetics, vol. 16, no. 14, pp. 1661-1675, 2007.

[61] T. P. Stossel, J. Condeelis, L. Cooley et al., "Filamins as integrators of cell mechanics and signalling," Nature Reviews Molecular Cell Biology, vol. 2, no. 2, pp. 138-145, 2001.

[62] H. Gao, W. Priebe, J. Glod, and D. Banerjee, "Activation of signal transducers and activators of transcription 3 and focal adhesion kinase by stromal cell-derived factor 1 is required for migration of human mesenchymal stem cells in response to tumor cellconditioned medium," Stem Cells, vol. 27, no. 4, pp. 857-865, 2009.

[63] J. Y. Hsieh, T. S. Huang, S. M. Cheng et al., "miR-146a-5p circuitry uncouples cell proliferation and migration, but not differentiation, in human mesenchymal stem cells," Nucleic Acids Research, vol. 41, no. 21, pp. 9753-9763, 2013.

[64] T. Ponomaryov, A. Peled, I. Petit et al., "Induction of the chemokine stromal-derived factor-1 following DNA damage improves human stem cell function," Journal of Clinical Investigation, vol. 106, no. 11, pp. 1331-1339, 2000. 
[65] T. Hung and H. Y. Chang, "Long noncoding RNA in genome regulation: prospects and mechanisms," RNA Biology, vol. 7, no. 5, pp. 582-585, 2010.

[66] H. Ma, Y. Hao, X. Dong et al., "Molecular mechanisms and function prediction of long noncoding RNA," TheScientificWorldJOURNAL, vol. 2012, Article ID 541786, 11 pages, 2012. 

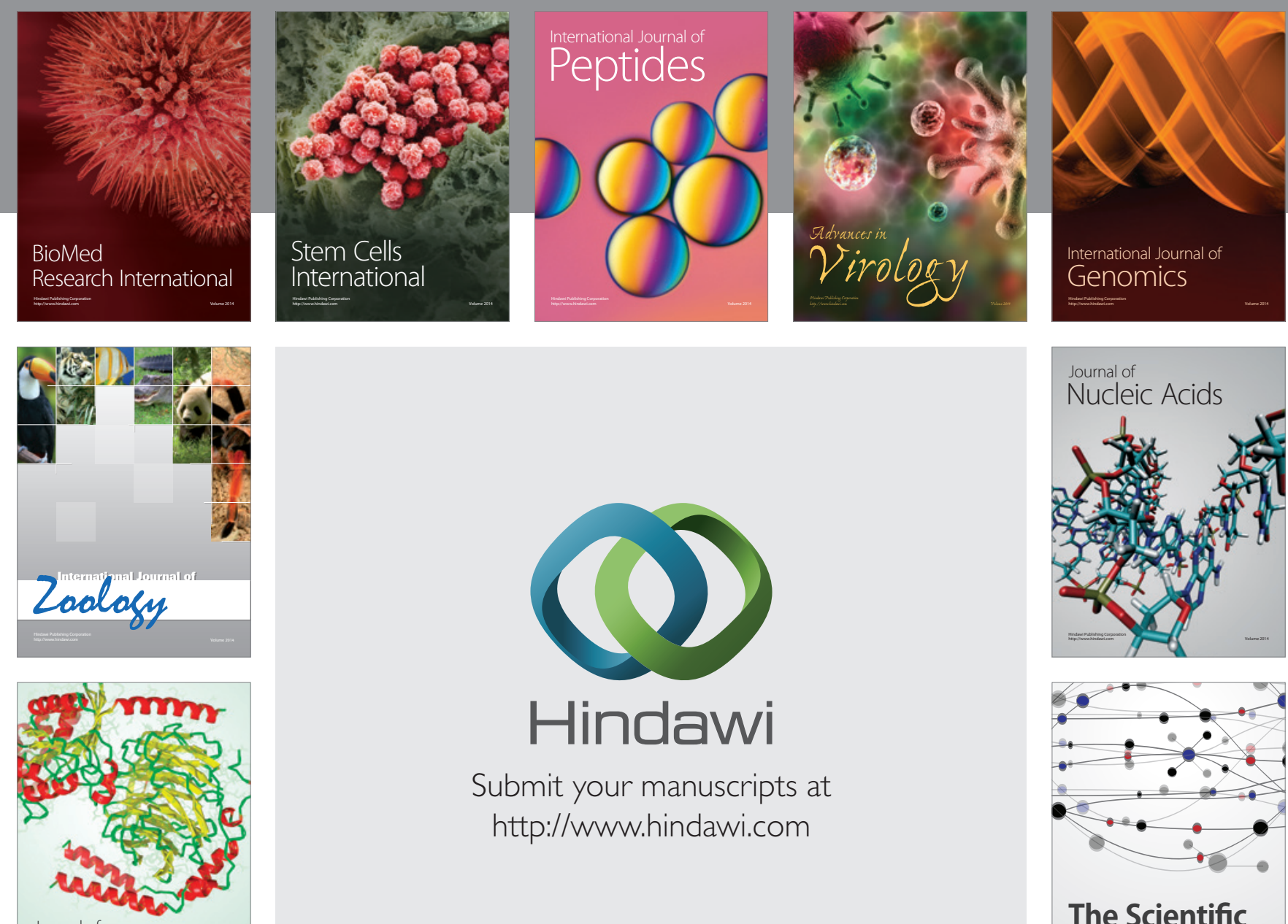

Submit your manuscripts at

http://www.hindawi.com

Journal of
Signal Transduction
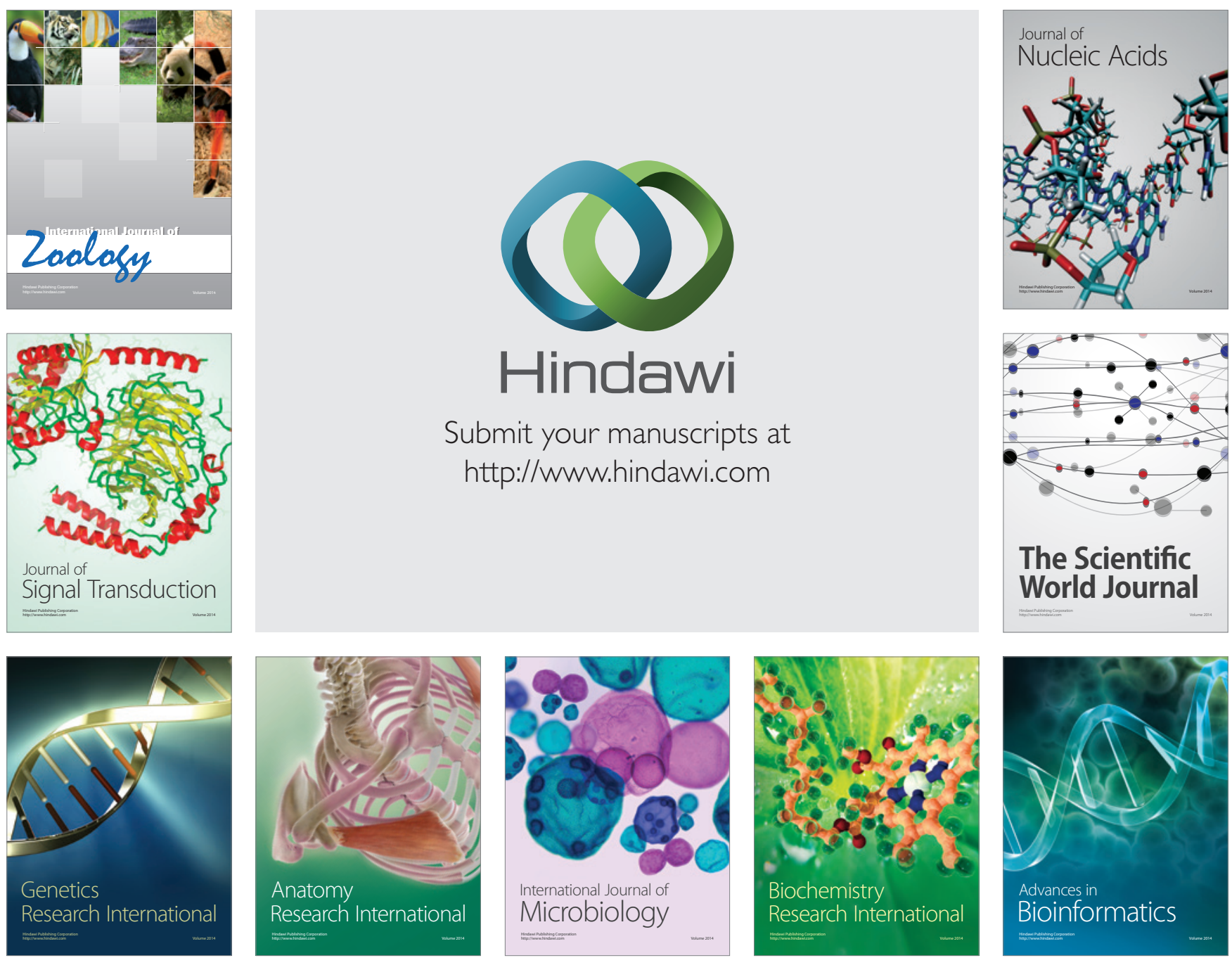

The Scientific World Journal
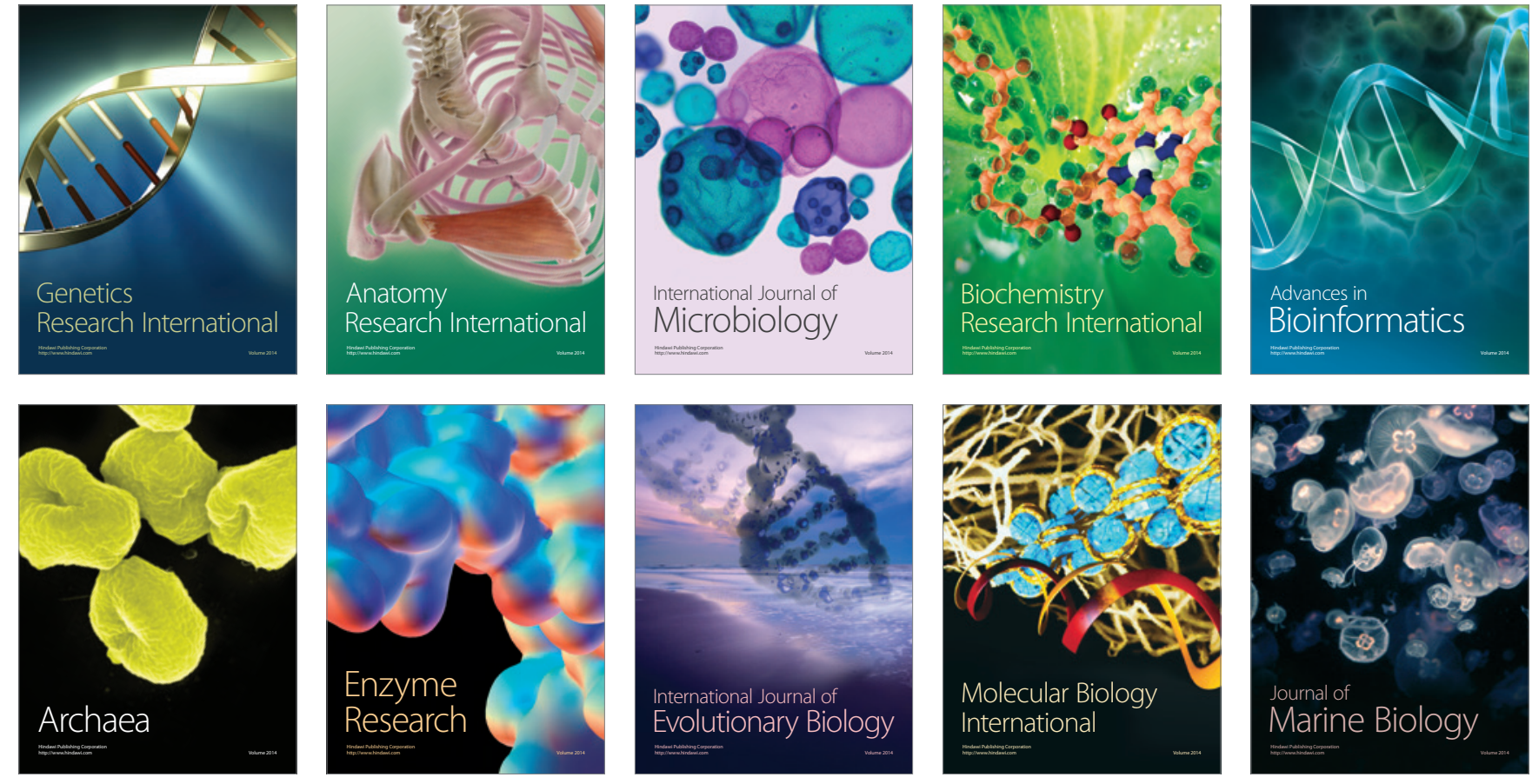